\title{
Selective Epitaxial Growth of Sub-micron Structures of YBaCuO by Substrate Modification
}

\author{
Dave H.A. Blank, Cas A.J. Damen, Boike L. Kropman, and Horst Rogalla \\ Department of Applied Physics, Low Temperature Division, University of Twente, \\ P.O.Box 217, 7500 AE Enschede, The Netherlands
}

Sub-micron structures of high-Te thin films have been realized with Selective Epitaxial Growth (SEG). Two different techniques to achieve SEG have been studied. First, narrow trenches down to $100 \mathrm{~nm}$ are etched into the substrate with a four-layer E-beam lithography technique. Second, amorphous metal layers have been used to define pattern definition masks. Besides the suitability of both techniques, also the potential to combine these techniques is part of this study.

\section{INTRODUCTION}

Superconducting properties of sub-micron structures of high-Tc thin films deteriorate due to the patterning techniques used. This deterioration is, e.g., caused by oxygen outdiffusion during (reactive) ion etching by local heating or by reactions of the high-Tc thin film with resist and chemicals, needed for the lithographic processes. Selective Epitaxial Growth (SEG), where no post-deposition patterning is necessary, offers new possibilities.

In this study two different techniques to achieve SEG are presented. First, narrow trenches down to $100 \mathrm{~nm}$ are etched into the substrate with a fourlayer E-beam lithography technique. With this method a pattern etched by $\mathrm{SF}_{6}$ and $\mathrm{O}_{2}$ in a $\mathrm{Au} / \mathrm{Ti}(\mathrm{Ox})$-thin film on top of an AZ-resist layer is transferred into the substrate by Ar-etching. Second, the pattern needed is defined by locally changing the crystallographic properties of the substrate with pattern definition masks of $\mathrm{Ti}$, used as a reactive agent for local chemical or crystallographic modification of the overlying layer.

\section{SELECTIVE EPITAXIAL GROWTH}

\subsection{Substrate-etching}

To reduce the problems of direct etching of a high-Tc thin film, one can etch the less (temperature) sensitive substrate. Steep and welldefined trench-edges are essential for this technique, because there must be no (or bad) electrical contact between in- and outside of the trench. De Nivelle [1] deposited niobium onto a substrate and structured it by a 3-layer EBL (E-beam lithographic) resist method. The Nb-layer served as a mask to structure the substrate by Ar-IBE. Afterwards, the $\mathrm{Nb}$ has to be removed by $\mathrm{SF}_{6}$-RIE followed by an annealprocedure.

Here, we present an other approach using titanium as an etch-stop. $\mathrm{Ti}$ is one of the most resistant materials in Ar-based etching methods as well as in $\mathrm{O}_{2}$-RIE. On the other hand, the etching of $T i$ itself can be very difficult due to the reactivity of a 'clean' titanium surface, even at very low background pressures (as low as $10^{-6}$ Torr). To overcome this problem, the following procedure was used.

On a $\left(\mathrm{SrTiO}_{3}\right)$ substrate we spin-coated a bottom resist layer (500 nm AZ1518 or PMMA) and prebaked it at $120^{\circ} \mathrm{C}$. Afterwards a $40 \mathrm{~nm}$ thin Tilayer is sputter deposited on the resist, in situ followed by a deposition of a $20 \mathrm{~nm}$ thin Au-film. The latter protects the titanium from oxidation. Finally, a thin E-beam sensitive resist is spun onto the metallic interlayers.

From extensive studies we achieved the following etch process parameters. Efficient etching of $\mathrm{Ti}$ in $\mathrm{SF}_{6}$-RIE only takes place at low pressure $\left(<2 \times 10^{-3}\right.$ mbar), admixing oxygen did not result in an increase of the fluorine concentration, and the etch-rate is very sensitive to the exposed area. For small areas $\left(<1 \mathrm{~mm}^{2}\right)$ the etch-rate stabilized on $6 \mathrm{~nm} / \mathrm{min}$ at a pressure of $1.8 \times 10^{-3}$ mbar and a self-bias of $-120 \mathrm{~V}$.

The anisotropic resist etching by oxygen-RIE is 
very sensitive to process parameters. Although oxygen plasmas are routinely applied to strip resist layers, lateral anisotropic etching is still under study. Good results are obtained by baking the resist at higher temperatures. In our case we want to avoid this, because it limits our lift-off process. Best results were obtained with high $\mathrm{O}_{2}$ gas flow (14 sccm), input power of $100 \mathrm{~W}\left(0.32 \mathrm{~W} / \mathrm{cm}^{2}\right)$, selfbias of $-300 \mathrm{~V}$ and pressure of $1.5 \times 10^{-2} \mathrm{mbar}$.

Finally, the pattern is transferred into the substrate by Ar-etching with the " $\mathrm{MiO}$ on top of the resist-Au-Ti-layer as an etch-stop. Afterwards, the resist is removed by lift-off, so no damaging of the substrate-surface takes place and no post-anneal is necessary.

\subsection{Definition masks}

With standard optical or E-beam lithography a sputter-deposited $10 \mathrm{~nm}$ thin Ti layer is structured by lift-off. Next, a $80 \mathrm{~nm}$ thin YBCO layer is deposited on the entire substrate. Due to the high deposition temperature and oxygen atmosphere, the Ti-layer will oxidize, forming $\mathrm{TiO}_{\mathrm{x}}$. The diffusion of the $\mathrm{Ti}$ is expected to be less, compared to other materials used in literature, like $\mathrm{Si}$ or $\mathrm{W}[2,3]$. The diffusion, especially in lateral direction, is the limiting factor is this SEG-process.

Several techniques were used to verify the mechanism that is responsible for ruining the superconducting properties on top of the metal mask. In Figure 1, a crater edge Auger profile of the substrate-Ti-YBCO interface is shown

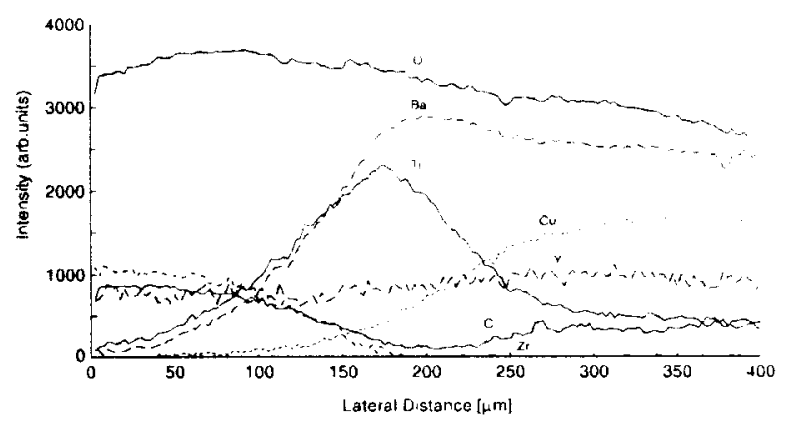

Fig. 1 Crater-edge profile of YBCO on Ti-YSZ.

The shown depth-profile is taken as a linescan along the edge of an under grazing angle etehed crater. From this profile it can be seen that $T_{1}$ diffuses only to a small extent into the YBCO-layer. On the other hand, $\mathrm{Ba}$ diffuses into the entire Tilayer, likely forming $\mathrm{BaTiO}_{3}$. This leads to a $\mathrm{Ba}$ defieiency in the first YBCO-layers. SEM pictures show a large density of grains and XRD reveals only $c$-axis orientations, although in a much lesser extent than the non-covered substrate, indicating a layer with small crystallites. The layer shows a semiconducting (and by that isolating) behaviour, with a resistivity $\rho(100 \mathrm{~K})=56.5 \Omega \mathrm{cm}$.

The electrical properties $(\rho$, and IVcharacteristics) of a bridge, made with these structuring technique, is given in figure 2. The submicron structure is a bridge of $600 \mathrm{~nm}$ wide and 300 $\mathrm{nm}$ long. The 'zero temperature' (Tc) of the bridge is $88 \mathrm{~K}$ and the critical current density $\mathrm{J}_{\mathrm{c}}=2.7 \times 10^{6}$ $\mathrm{A} / \mathrm{cm}^{2}$

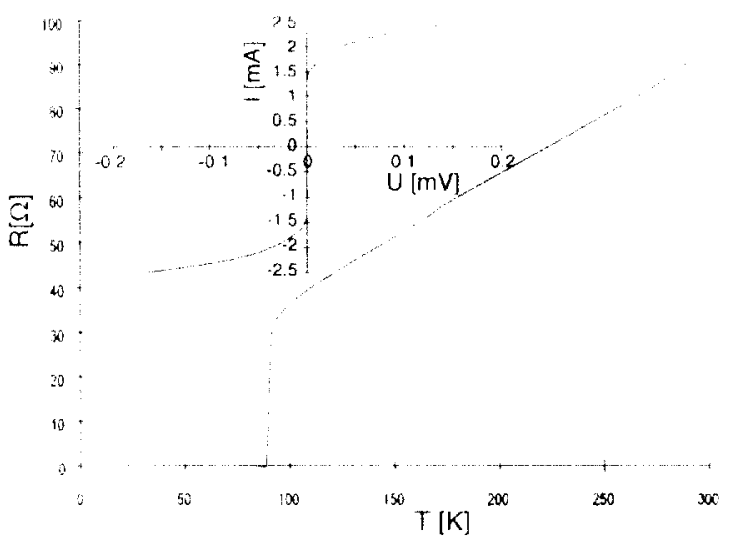

lig. 2. IV-curve and $R$ versus $T$ for sub-micron structured YBCO bridge

From these measurements we can conclude that the diffusion of $\mathrm{Ti}$ into the $\mathrm{YBaCuO}$ layer is negligible. This restricts this SEG technique not only to planar structures but makes it also useful in multilayer designs.

A combination of both presented techniques is feasible due to the lift-off possibility after substrate elching

\section{REFERENCES}

1 De Nivelle et al., to be published in Physica $C$ 2. Q.Y. Ma et al., Appl. Phys. Lett., 55 (1989) 896. 3. D.H.A. Blank et al., proc. EUCAS (1993) 675. 